and iron in powdered form for use at high frequencies in various types of magnetic loading and radio cores, it is inevitable that high degrees of lattice strain are introduced and this must be reduced to a minimum to obtain the magnetic characteristics required. By examining the resolution of the lines on the $\mathrm{X}$-ray patterns of powders after heat treatment at successively increasing temperatures, it is possible to determine at what stage the strain is eliminated, and so an appropriate 'annealing' schedule can be chosen. Moreover, by the examination of finished powder, variations in the manufacturing conditions are quickly revealed.

The examples given here are but a random selection from the very many and varied applications of X-ray technique. They should, however, enable some picture to be formed of the extensive manner in which X-ray methods may be utilized in the industrial laboratory.

${ }^{1}$ Mechanical Engineering, p. 906, Dec., 1941.

¿ Seemann, H. E., Proc. Amer. Soc. Testing Materials, 38, 284 (1938). ${ }^{3}$ Bradley, A. J., and Jay, A. H., Proc. Phys. Soc., 44, 563 (1932).

4 Lipson, H., and Wilson, A. J. C., J. Sei. Inst., 18, 144 (1941).

-Bradley, A. J., Bragg, W. L., and Sykes, C., J. Iron and Steel Inst., $141,63(1940)$.

- Lipson, H., Nature, 146, 798 (1940).

\section{MEASUREMENT OF RADIATION FOR MEDICAL PURPOSES*}

\author{
BY PROF. W. V. MAYNEORD \\ Royal Cancer Hospital, London
}

$\mathrm{T}$ HE methods of measuring radiation for any purpose must in the end be determined by the fact that we are concerned with a form of energy. In particular, the biological or medical use of radiation is dominated by the rule that absorbed energy alone is effective, so that we are forced in considering the physical aspects of the use of radiations for these purposes to look very closely into the measurement of absorbed energy.

Considering the case of $X$ rays first of all, it has for many years been recognized that the most promising basis on which to construct a system of measurement is the ionization produced by them in air, and since 1928 the röntgen based upon this finding has been used as the internationally accepted unit of dose. In 1937, as the result of a good deal of experimental and theoretical work, the use of the unit was extended to gamma rays, and a new definition of the röntgen was promulgated. The fundamental idea, namely, that we take all the electrons set free by the radiation from a certain mass of air, utilize as much as possible of their energy in producing ionization in air and measure the number of electrostatic units of charge thus liberated, appears much more clearly in the new definition.

Having in this way obtained a suitable unit, it was natural to apply it to medical problems, in particular to that of finding the dose at any point throughout the tissues of the body. First of all, the distribution of radiation along the axis of a beam of $X$ rays was studied over a very wide range of conditions from $10 \mathrm{kv}$. up to some $2,000 \mathrm{kv}$., and so a large amount of useful information was collected

* Substance of a lecture delivered to the Physical Society on Feb. 27. concerning the effective penetration of the beams. Later the studies were extended to include the complete three-dimensional problems of distribution and the study of 'isodose surfaces'. Simple geometrical methods of dealing with these problems were demonstrated, and it is possible by the use of plaster casts to estimate fairly closely the dose received at any given point within any arrangement of beams of radiation, however inclined or concentrated. Alternatively, the best arrangement of beams of radiation in order to produce the maximum dose at the point desired while at the same time keeping down the dose to normal healthy tissue, may be investigated.

So far we have been concerned with the amount of radiation at a particular element of volume, but it is important to develop this study so as to estimate the total energy absorbed by the body as a whole, the estimates of energy absorption to be made if possible in absolute energy units.

It will be recognized that the röntgen is essentially a unit of energy absorption in air, the factor of proportionality depending upon the energy required to produce one ion pair. If this energy is 33 electron. volts, it follows that when 1 röntgen is delivered to $1 \mathrm{gm}$. of air there will be an absorption of energy of about 85 ergs. For soft animal tissues the value will vary a little with wave-length, but for many purposes may be assumed to be equal to this amount. For the further development of the theory it is also necessary to consider the relationship between the intensity of a beam of $\mathbf{X}$ or gamma rays in ergs $\mathrm{cm}^{2}$.sec. and the dosage-rate, that is, r./min. Evidently the relationship depends upon the wave-length of the radiation, and it is easily calculated that whereas the energy flux per $\mathrm{cm}^{2}$. per röntgen for gamma rays is of the order of 3,000 ergs, for long wave-lengths of the order of $1 \mathrm{~A}$. the corresponding number is about 30 ergs. The distinction between intensity and dosage-rate is a very important one, which is all too frequently neglected in theory and practice.

In considering some of the effects of radiation, it seems that the total energy absorbed throughout the body is a very important quantity. The distribution studies of dose, the isodose surfaces, coupled with the deduction of the energy absorbed per gm. per röntgen, enable estimates of the energy absorbed to be made. We have used as a unit the energy absorbed when 1 röntgen is given to $1 \mathrm{gm}$. of air, and called it 1 gram-röntgen. In clinical practice a megagram-röntgen is of a convenient order of size and may be shown very easily to be approximately 2 gm.cal.

Again, by geometrical and analytical methods (assuming simple laws of variation of radiation with depth) the integration of the energy absorbed throughout any part of the body may be carried out, and so the total energy absorbed may be estimated. This has been done for a number of conditions occurring in practice and it is found that the physical results are very instructive in relation to the clinical findings. As an example of the kind of problem in which the results are significant, we may quote the problems of the protection of staff and patients from unwanted radiation, where the differences now revealed in the total energy absorbed in the body as a whole per unit dose on the skin raise the question of the assumed independence of 'tolerance dose' of wave-length. By the use of large numbers of pressed bakelite - carbon condenser ionization chambers throughout a model of the body, this subject is being investigated further. 
Having obtained estimates of the energy flux into the body and of the energy absorbed from beams of $X$ and gamma rays, it is natural to attempt the corresponding solution for ultra-violet and infra-red rays. By the use of calibrated thermocouples, information on this subject may be obtained, and indeed in the case of ultra-violet light a good deal is already available. It seems that the total energy absorbed per square centimetre of the body when irradiated with ultra-violet light so as to produce a threshold erythema is of the same order as that given when $100 \mathrm{r}$. of medium wave-length $\mathrm{X}$ rays is administered. For infra-red radiation very little information was available, but we have used suitably mounted calibrated vacuum thermocouples (General Electric type) and made measurements of the intensities required for a painful sensation and of the intensities normally employed in therapy. The values of these quantities are found to vary with the colour temperature of the source, the normal intensity for a 500-watt tungsten filament lamp in a reflector being of the order of $1.5 \mathrm{gm} . \mathrm{cal} . / \mathrm{cm}^{2} . \mathrm{min}$.

Finally, then, we see the order of intensities and amounts of energy involved in the use of different kinds of radiations.

(a) Type of radiation.

High-voltage X rays. $200 \mathrm{kv} . \quad 40 \mathrm{r} . / \mathrm{min} . \quad 2 \times 10^{3}$

Heavy fliter

Ultra-violet light

$20 \mu \mathrm{watts} / \mathrm{cm} .^{2}$

$2 \times 10^{3}$

Infra-red

$1 \mathrm{gm} . \mathrm{cal} . / \mathrm{cm} .{ }^{2} \mathrm{~min}$.

$7 \times 10^{5}$

(b) Type of radiation

High-voltage $\mathbf{X}$ rays

rays $200 \mathrm{kv} . \quad 30$ megagram- $\quad 60$ gm.cal. in

Total energy absorbed

Ultra-violet light röntgens.

6 weeks.

Erythema over 8 gm.cal. at body surface of a sitting. body surface

Infra-red

$1 \mathrm{gm} . \mathrm{cal} . / \mathrm{cm} .{ }^{2} \mathrm{~min} .3 \times 10^{4} \mathrm{gm}$. 20 min. cal. at a sit Area 1,500 $\mathrm{cm}^{2}$. ting.

The intensities are approximate but of interest in the correlation of all the radiations on an energy basis.

\section{OXIDATION OF HYDROCARBONS AT LOW TEMPERATURES}

\section{By P. GEORGE, PROF. E. K. RIDEAL, F.R.S., and A. ROBERTSON \\ Department of Colloid Science, Cambridge}

$\mathrm{A}^{\mathrm{A}}$ ARMER and Sundralingam in a recent paper ${ }^{1}$ have stressed the part that hydroperoxides play as the first isolatable intermediates in autoxidations : their investigation, anticipated by Criegee, showing that olefin peroxides have the structure $-\mathrm{CH}(\mathrm{OOH})-\mathrm{CH}=\mathrm{CH}-$, brings these peroxides into line with Rieche's alkyl peroxides, possessing the reactive group ( $-\mathrm{OOH})$. The work reported below on the uncatalysed and the heavy-metal catalysed oxidation of alkyl benzenes and long-chain saturated aliphatic hydrocarbons $\left(\mathrm{C}_{15}\right.$ to $\left.\mathrm{C}_{25}\right)$ in the liquid phase at $100-120^{\circ} \mathrm{C}$., also supports the hypothesis that hydroperoxides are primary oxidation intermediates.

\section{The Chain Character of the Reaction}

In the case of alkyl benzenes the peroxide found after reaction corresponds to $60-80$ per cent of the oxygen absorbed. The peroxides of long-chain paraffins are not so stable; and they are only found in small concentration, about 5 per cent. In the metal-catalysed oxidation good agreement is obtained between the observed peroxide concentration and that calculated from the efficiency of the catalyst in peroxide decomposition.

In the catalysed oxidations a curious independence of oxidation-rate of catalyst concentration was found above a certain value. This has been reported in other autoxidations, notably benzaldehyde catalysed by ferrous phthalocyanine ${ }^{2}$ and linseed oil catalysed by hæmoglobin ${ }^{3}$. The explanation advanced was low oxygen availability due to physical conditions. This cannot be the case here, for the rate of oxidation has been found proportional to the partial pressure of oxygen. The curves obtained for longchain paraffins catalysed by cobalt stearate and ethyl benzene catalysed by copper stearate are shown in Fig. 1.

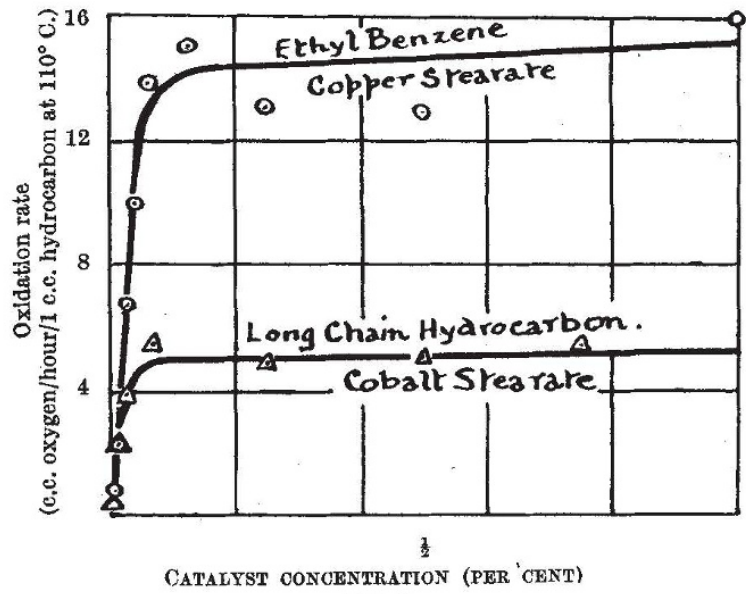

Fig. 1.

This can be explained by the hypothesis of a chain mechanism in which the metallic catalyst both starts and stops chains. This gives the expression:

$$
\text { Rate of oxidation }=\frac{a+b Y}{c+d Y}
$$

$Y$ is the catalyst concentration, $a$ the number of chains started per unit time in the uncatalysed reaction, $b$ the number started per catalyst molecule $1 / c$ and $1 /(c+d Y)$ the uncatalysed and catalysed chain lengths respectively.

The chain character of the oxidation has been demonstrated by inhibition with $\beta$-naphthol. It causes a marked diminution in the oxidation rate of alkyl benzenes, the rate slowly increasing with time. For catalysed long-chain paraffins complete induction periods lasting for 2-6 hours with no measurable oxygen uptake are observed, even with a 2-50 molar excess of catalyst over inhibitor. Equations have been developed to calculate the chain length from this diminished rate or induction period data. A surprising specificity of catalysts is shown by the following experimental results :

(a) Copper stearate catalyses the oxidation of ethyl benzene and tetralin, but not long-chain 